\title{
Comparison of enterotoxins and haemolysins produced by methicillin-resistant (MRSA) and sensitive (MSSA) Staphylococcus aureus
}

\author{
J. E. COIA, LYNDA BROWNING, LORRAINE HAINES, T. H. BIRKBECK* and D. J. PLATT \\ University Department of Bacteriology, 94 Castle Street, Glasgow G4 OSF and Department of Microbiology, University \\ of Glasgow, Glasgow G12 800
}

\begin{abstract}
Summary. A collection of 201 isolates of Staphylococcus aureus was examined : 152 methicillinsensitive $S$. aureus (MSSA) comprised 48 blood culture isolates (BC) and 58 isolates from routine diagnostic specimens (RD) from Glasgow Royal Infirmary (GRI), and 46 strains from nasal swabs of patients attending a general practitioner (GP); 49 isolates were of methicillin-resistant $S$. aureus (MRSA) from GRI. We have previously shown that the MRSA could be divided into two sub-groups on the basis of sensitivity or resistance to aminoglycoside antibiotics. Production of enterotoxins A, B, C and D, and $\alpha-, \beta-, \gamma$ - and $\delta$ haemolysins was detected by reverse passive latex agglutination (RPLA) and agar overlay methods respectively: $60 \%$ of BC MSSA and a similar proportion of MSSA from other sources produced enterotoxin $; 87 \%$ of aminoglycoside-sensitive MRSA produced enterotoxin ( $89 \%$ of these produced enterotoxin A alone) whereas only $27 \%$ of aminoglycoside-resistant MRSA were enterotoxin-positive, significantly less than either MSSA or aminoglycosidesensitive MRSA. The proportion of haemolysin-producing isolates did not differ amongst the isolates of MSSA and MRSA; there was no difference in the distributions of haemolysins between aminoglycoside-sensitive and -resistant strains of MRSA. GP MSSA had higher and lower numbers of $\gamma$ - and $\delta$-haemolysin producers respectively than other $S$. aureus isolates. $\alpha$-Haemolysin producers were commoner amongst MRSA isolates, which were also more likely than MSSA isolates to produce several haemolysins. Differences in enterotoxin production between aminoglycoside-sensitive and -resistant MRSA isolates reflect subgroups previously defined by biotype, phage type, immunoblot and restriction enzyme fragmentation pattern data, and provide further evidence for the existence of two major MRSA clones in GRI.
\end{abstract}

\section{Introduction}

Staphylococcus aureus infections are important causes of morbidity and mortality in hospitals and the community. The spectrum of disease produced by these organisms and the range of pathogenic processes is very wide and includes toxin-mediated phenomena, direct tissue destruction and secondary immune mechanisms. ${ }^{1,2}$ In recent years the therapy of such infections has been complicated by the widespread occurrence of methicillin-resistant $S$. aureus (MRSA) isolates. ${ }^{3,4}$ MRSA have been responsible for outbreaks of nosocomial infection world-wide, ${ }^{5}$ especially in Australia ${ }^{6,7}$ North America ${ }^{8}$ and, more recently, in many parts of Europe, including Ireland ${ }^{9,10}$ and Great Britain, ${ }^{5,11}$ and significant numbers of isolates have been reported from a number of other countries, including Greece, France, Portugal, Italy and the Middle East. ${ }^{11-13}$
The pathogenic potential of MRSA, in comparison with methicillin-sensitive $S$. aureus (MSSA), has been the subject of controversy and speculation. ${ }^{5.12}$ The production of various determinants of pathogenicity, including protein $\mathrm{A}$ and coagulase,${ }^{15-17}$ lipase, ${ }^{18}$ haemolysin ${ }^{17,19}$ and enterotoxin ${ }^{17,19,20}$ has been investigated.

We have shown previously that community isolates of MSSA and hospital MSSA and MRSA isolates may be characterised by means of plasmid profiles and restriction enzyme fragmentation patterns (REFP) ${ }^{21}$ We have also demonstrated that MRSA isolates in Glasgow Royal Infirmary (GRI) comprise two major clones on the basis of phage type, biotype, immunoblot pattern and REFP. ${ }^{22}$ In this study we have extended the analysis of these strains to include pathogenicity factors by comparing the production of enterotoxins and haemolysins by reverse passive latex agglutination and agar overlay methods, respectively.

Received 28 Feb. 1991 ; accepted 19 April 1991. 


\section{Materials and methods}

\section{Organisms}

A collection of 201 isolates of $S$. aureus was studied : 49 MRSA isolates, 48 consecutive MSSA isolates from blood cultures (BC) and 58 MSSA isolates from other routine diagnostic specimens (RD) from GRI, as well as 46 MSSA isolates from the noses of patients attending a general practitioner (GP). Multiple isolates from the same patient were excluded. All were identified as $S$. aureus on the basis of their colonial morphology and positive results in coagulase and latex agglutination (Staphaurex, Wellcome) tests. ${ }^{21}$

The MRSA isolates were divided into two groups on the basis of sensitivity or resistance to various aminoglycoside antibiotics. ${ }^{21}$ We have shown previously that this difference in antibiogram reflects the existence of two major MRSA clones in GRI as defined by phage type, biotype, immunoblot and REFP. ${ }^{22}$

\section{Enterotoxin production}

Production of enterotoxins A, B, C and D was determined by reverse passive latex agglutination (RPLA), with commercial kit tests (SET-RPLA, Oxoid), according to the manufacturer's instructions. ${ }^{19}$ Isolates were inoculated on to nutrient-agar plates and incubated for $24 \mathrm{~h}$ at $37^{\circ} \mathrm{C}$. Sufficient growth was harvested from these plates to provide $c$. $2 \mu \mathrm{g}$ bacterial dry weight $/ \mathrm{ml}$; this was inoculated into $10 \mathrm{ml}$ of nutrient broth and incubated for $18 \mathrm{~h}$ at $37^{\circ} \mathrm{C}$ in an orbital incubator. After centrifugation at $2000 \mathrm{~g}$ for $15 \mathrm{~min}$ at $4^{\circ} \mathrm{C}$, the upper $10 \mathrm{ml}$ of supernate was retained for analysis.

Into each well of five rows of a V-bottomed microtitration plate, $25 \mu$ lof phosphate-buffered saline containing bovine serum albumin $0.5 \% \mathrm{w} / \mathrm{v}$ was dispensed and $25 \mu \mathrm{l}$ of the test culture supernate was then dispensed into the first well of each row and doubly diluted; the dilutions were not extended into the last well of each row. To each well of the first row was added $25 \mu$ l of latex sensitised with rabbit antistaphylococcal enterotoxin A IgG antibodies; to row $2,25 \mu \mathrm{l}$ of anti-enterotoxin-B-sensitised latex; to row $3,25 \mu$ l of anti-enterotoxin-C-sensitised latex; to row 4, $25 \mu$ l of anti-enterotoxin-D-sensitised latex; and to row $5,25 \mu \mathrm{l}$ of a control latex suspension coated with non-immune rabbit globulin. The contents were mixed thoroughly by micromixer, covered, and left undisturbed on a vibration-free surface at room temperature for 20-24 h. Each of the test wells (rows 1-4) was then examined for agglutination which indicated the presence of the respective enterotoxin (A, B, C or D). The absence of agglutination in row 5 and in the last well of rows 1-5 (negative controls) served to exclude spurious non-specific agglutination and auto-agglutination, respectively.

\section{Detection of haemolysins}

Production of $\alpha-, \beta$-, $\gamma$ - and $\delta$-haemolysins was detected by a modification of methods described elsewhere. ${ }^{19,23}$ Rabbit anti- $\alpha$ antiserum and $\delta$-haemolysin were supplied by Dr T. H. Birkbeck, Department of Microbiology, University of Glasgow. Five colonies of each test organism were inoculated into $1-\mathrm{ml}$ volumes of brain-heart infusion broth and incubated for $18 \mathrm{~h}$ at $37^{\circ} \mathrm{C}$ in air; these cultures were then inoculated in batches of 20 , with a multi-point apparatus, on to three nutrient-agar plates for detection of $\alpha-, \beta$ - and $\delta$-haemolysin. For $\gamma$-haemolysin production, the cultures were inoculated on to a nutrient-agarose plate. The plates were incubated for $18 \mathrm{~h}$ at $37^{\circ} \mathrm{C}$ in air.

$\alpha$-Haemolysin production. The nutrient-agar plates were overlaid with $5 \mathrm{ml}$ of rabbit blood $5 \% \mathrm{v} / \mathrm{v}$ agar. After the agar had set, 2-mm diameter wells were cut in the spaces between the test strains. Into these wells were dispensed $5 \mu \mathrm{l}$ of rabbit anti- $\alpha$-haemolysin antiserum. After incubation for $24 \mathrm{~h}$ in air at $37^{\circ} \mathrm{C}$, the plates were examined for haemolysis, with specific inhibition of $\alpha$-haemolysin activity around the wells containing antiserum.

$\beta$-Haemolysin production. The nutrient-agar plates were overlaid with $5 \mathrm{ml}$ of sheep blood $5 \% \mathrm{v} / \mathrm{v}$ agar. After the agar had set, 2-mm diameter wells were cut in the spaces between the test strains. Into these wells were dispensed $5 \mu \mathrm{l}$ of $\delta$-haemolysin. After incubation for $24 \mathrm{~h}$ in air at $37^{\circ} \mathrm{C}$, the plates were examined for haemolysis due to $\beta$-haemolysin, which could be recognised by enhancement around the wells containing $\delta$-haemolysin.

$\gamma$-Haemolysin production was determined in a manner similar to that used for $\alpha$-haemolysin. The nutrientagarose plates were overlaid with $5 \mathrm{ml}$ of rabbit blood $5 \% \mathrm{v} / \mathrm{v}$ agarose. After the agarose had set, 2-mm diameter wells were cut in the spaces between the test strains. Into these wells were dispensed $5 \mu \mathrm{l}$ of rabbit anti- $\alpha$-haemolysin serum. After incubation for $24 \mathrm{~h}$ in air at $37^{\circ} \mathrm{C}$, the plates were examined for haemolysis; spurious $\alpha$-haemolysin activity was inhibited by the antiserum.

$\delta$-Haemolysin production was determined on nutrient-agar plates overlaid with $5 \mathrm{ml}$ of cod blood $5 \%$ $\mathrm{v} / \mathrm{v}$ agar. The plates were incubated for $4-8 \mathrm{~h}$ at $4{ }^{\circ} \mathrm{C}$ and observed for haemolysis.

\section{Statistical analysis}

The distribution of enterotoxins and haemolysins amongst the various groups of organisms was compared by means of the $\chi^{2}$ or Fisher's exact test as appropriate. $^{24}$

\section{Results}

\section{Enterotoxin production}

Table I and fig. 1 summarise the results of RPLA testing for enterotoxins A, B, C and D. The data are 
Table I. Distribution of enterotoxin production in isolates from different sources

\begin{tabular}{|c|c|c|c|c|c|}
\hline \multirow{2}{*}{$\begin{array}{l}S . \text { aureus } \\
\text { group }\end{array}$} & \multirow{2}{*}{$\begin{array}{l}\text { Number of } \\
\text { isolates }\end{array}$} & \multicolumn{4}{|c|}{$\begin{array}{c}\text { Number }(\%) \text { of isolates that } \\
\text { produced enterotoxin }\end{array}$} \\
\hline & & A & B & C & D \\
\hline BC & 48 & $17(35)$ & $8(17)$ & $9(19)$ & $7(15)$ \\
\hline GP & 46 & $20(43)$ & $4(9)$ & $16(35)$ & $6(13)$ \\
\hline RD & 58 & $18(31)$ & $8(14)$ & $5(9)$ & $4(7)$ \\
\hline $\operatorname{MRSA}(\mathrm{S})$ & 23 & $19(83)$ & $3(13)$ & $3(13)$ & $0(0)$ \\
\hline MRSA(R) & 26 & $5(19)$ & $3(12)$ & $4(15)$ & $0(0)$ \\
\hline
\end{tabular}

(S), Aminoglycoside sensitive; (R), aminoglycoside resistant.

shown separately for the MRSA isolates designated aminoglycoside-sensitive or -resistant, to demonstrate differences between them.

When the number of enterotoxin-A-producing isolates in each of the three MSSA sub-groups was compared, there was no significant difference ( $p>$ 0.5 ); for comparison with the MRSA isolates, these results were combined. The relative number of enterotoxin-A-producing isolates did not differ significantly between the MSSA (55 of 152) and aminoglycoside-resistant MRSA ( 5 of 26) groups. However, the aminoglycoside-sensitive MRSA isolates differed markedly from the others $-19(83 \%)$ of 23 isolates produced enterotoxin $A(p<0.001)$.

There was no significant difference $(p>0.5)$ in the distribution of enterotoxin-B-producing isolates between the three MSSA sub-groups, between the aminoglycoside-sensitive and resistant MRSA collections, or between the combined MSSA results ( 20 of 152 enterotoxin-B-producing) and the combined MRSA results (6 of 49 enterotoxin-B-producing).

No significant difference was found in the distribution of enterotoxin-C-producers amongst the $\mathrm{BC}$ and RD MSSA collections. The proportion of enterotoxinC-producers amongst these hospital MSSA strains (14 of 106) did not differ from that in the aminoglycosidesensitive ( 3 of 23) or -resistant (4 of 26) MRSA subgroups $(p>0 \cdot 5)$. However, significantly more enterotoxin-C-producing isolates (16 of 46) were found amongst the GP isolates than in either the combined $\mathrm{BC}$ and RD MSSA (14 of 106), or the combined MRSA $(7$ of 49$)(p<0.001)$.

There was no significant difference in the number of enterotoxin-D-producing isolates in any of the MSSA sub-groups ( $p>0 \cdot 5$ ), but none of the MRSA isolates produced enterotoxin $\mathrm{D}$ and the difference between the number of enterotoxin-D-producing isolates in the combined MSSA (17 of 152) and the MRSA ( 0 of 49) collections was significantly different $(\mathrm{p}<0.001)$.

Any isolate that produced one or more enterotoxin(s) was defined as an enterotoxin producer. There was no significant difference in the relative numbers of enterotoxin producers in the various MSSA collections $(p>0.5)$ (table II). There was a significantly greater proportion of enterotoxin-producers amongst the aminoglycoside-sensitive MRSA isolates (20 of 23) than in the combined MSSA ( 88 of 152) group ( $<<$

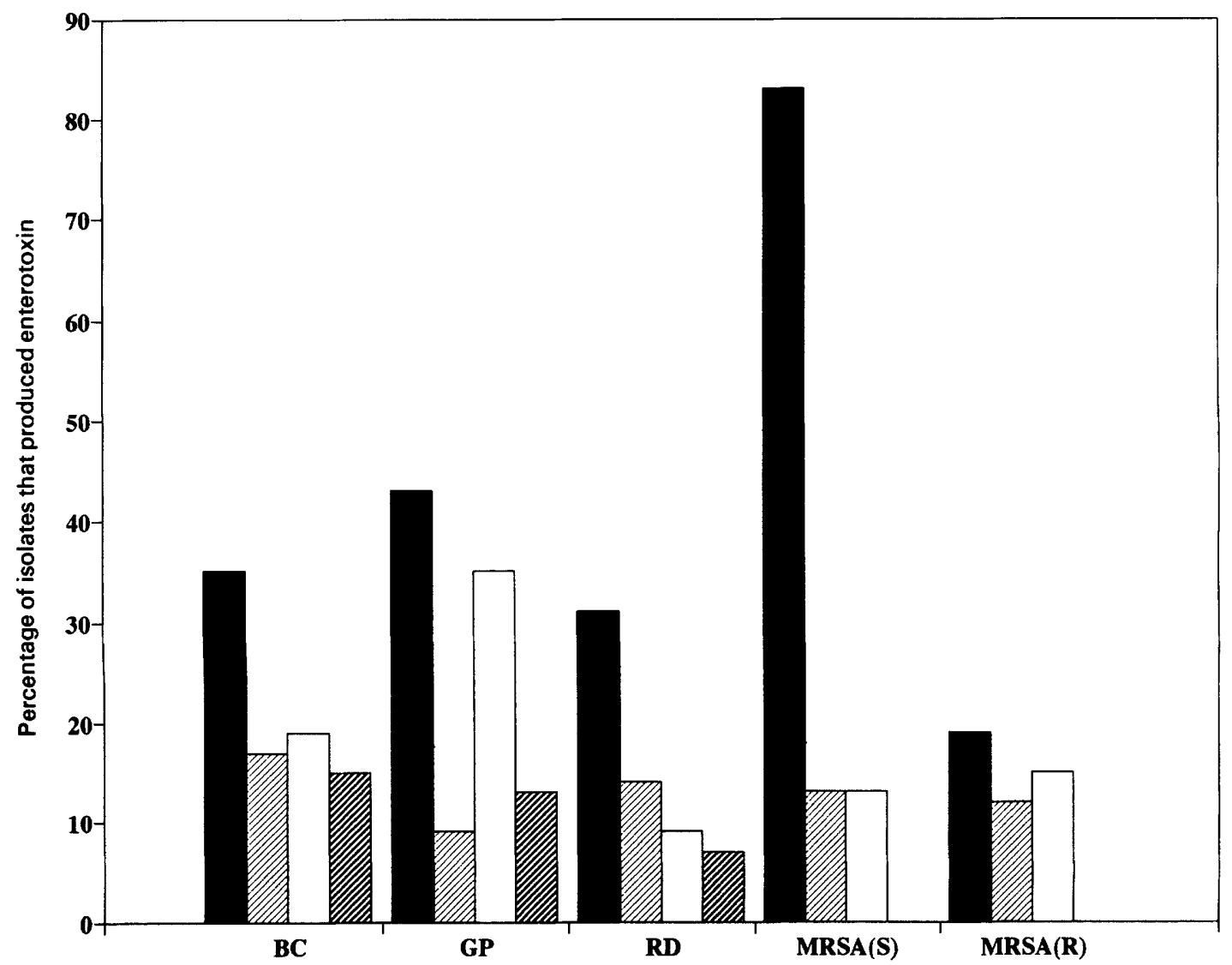

Fig. 1. Results of RPLA tests for enterotoxins of $S$. aureus: enterotoxin A $\mathbf{D}$; B $\square$;C $\square$;D 
Table II. Comparison of numbers of enterotoxin producers and non-producers amongst isolates from different sources

\begin{tabular}{cc|cc}
\hline & & $\begin{array}{c}\text { Number(\%) of isolates that were } \\
\text { enterotoxin } \\
\text { group }\end{array}$ & $\begin{array}{c}\text { Number of } \\
\text { isolates }\end{array}$ \\
\cline { 3 - 4 } & & producers & non-producers \\
\hline BC & 48 & $29(60)$ & $19(40)$ \\
GP & 46 & $29(63)$ & $17(37)$ \\
RD & 58 & $30(52)$ & $28(48)$ \\
MRSA(S) & 23 & $20(87)$ & $3(13)$ \\
MRSA(R) & 26 & $7(27)$ & $19(73)$ \\
\hline
\end{tabular}

Table III. Comparison of multiple enterotoxin production by isolates from different sources

\begin{tabular}{cc|rrr}
\hline & & \multicolumn{3}{|c}{$\begin{array}{c}\text { Number(\%) of isolates that } \\
\text { produced }\end{array}$} \\
$\begin{array}{ccccc}S . a u r e u s \\
\text { group }\end{array}$ & $\begin{array}{c}\text { Number of } \\
\text { isolates }\end{array}$ & \multicolumn{4}{|c}{$0^{*}$} & $1^{*}$ & $\geq 2^{*}$ \\
\cline { 3 - 5 } & & $19(39)$ & $21(44)$ & $8(17)$ \\
BC & 48 & $17(37)$ & $16(35)$ & $13(28)$ \\
GP & 46 & $38(48)$ & $26(45)$ & $4(7)$ \\
RD & 58 & $17(74)$ & $3(13)$ \\
MRSA(S) & 23 & $19(73)$ & $3(12)$ & $4(15)$ \\
MRSA(R) & 26 & & & \\
\hline
\end{tabular}

* Number of enterotoxins.

0.001). However, significantly fewer enterotoxin producers were found amongst the aminoglycosideresistant MRSA (7 of 26) than in either their aminoglycoside- or methicillin-sensitive counterparts $(\mathrm{p}<0.001)$.

When the distribution of enterotoxins was examined by subdividing the isolates according to the production of none, one, or two or more enterotoxins (table III), the BC and GP MSSA sub-groups gave similar results $(p>0 \cdot 5)$. However, relatively fewer of the RD isolates produced two or more enterotoxins with a concomitant increase in the number of single enterotoxin producers, although this difference only achieved statistical significance when compared with the GP isolates $(\mathrm{p}<$ 0.001).

Seventeen of the 20 enterotoxin-producing aminoglycoside-sensitive MRSA isolates produced a single enterotoxin; all 17 produced enterotoxin A. In contrast, although the aminoglycoside-resistant MRSA were predominantly non-producers, more than half of those that were enterotoxin-producers produced more than one enterotoxin. Thus, the pattern of enterotoxin production in aminoglycoside-sensitive and -resistant MRSA isolates differed markedly from that in the MSSA isolates $(p<0.001)$ but for different reasons; with the former group because of the preponderance of single enterotoxin-A-producers, the latter group as a result of the large number of non-producers (fig. 2).

\section{Haemolysin production}

Table IV and fig. 3 summarise the results of tests to detect the production of $\alpha-, \beta-, \gamma$ - and $\delta$-haemolysins. The distribution of MSSA isolates that produced $\alpha$ haemolysin did not differ between the BC, GP and RD sub-groups ( $p>0.5)$; therefore these were combined for comparison with MRSA isolates. Similarly, the relative numbers of $\alpha$-haemolysin producers amongst aminoglycoside-sensitive and resistant MRSA sub-groups did not differ ( $p>0.5$ ). However, significantly more of the combined MRSA isolates (36 of 49) produce $\alpha$-haemolysin than did the combined MSSA (86 of 152) isolates ( $p<0.001)$.

No significant difference was found in $\beta$-haemolysin

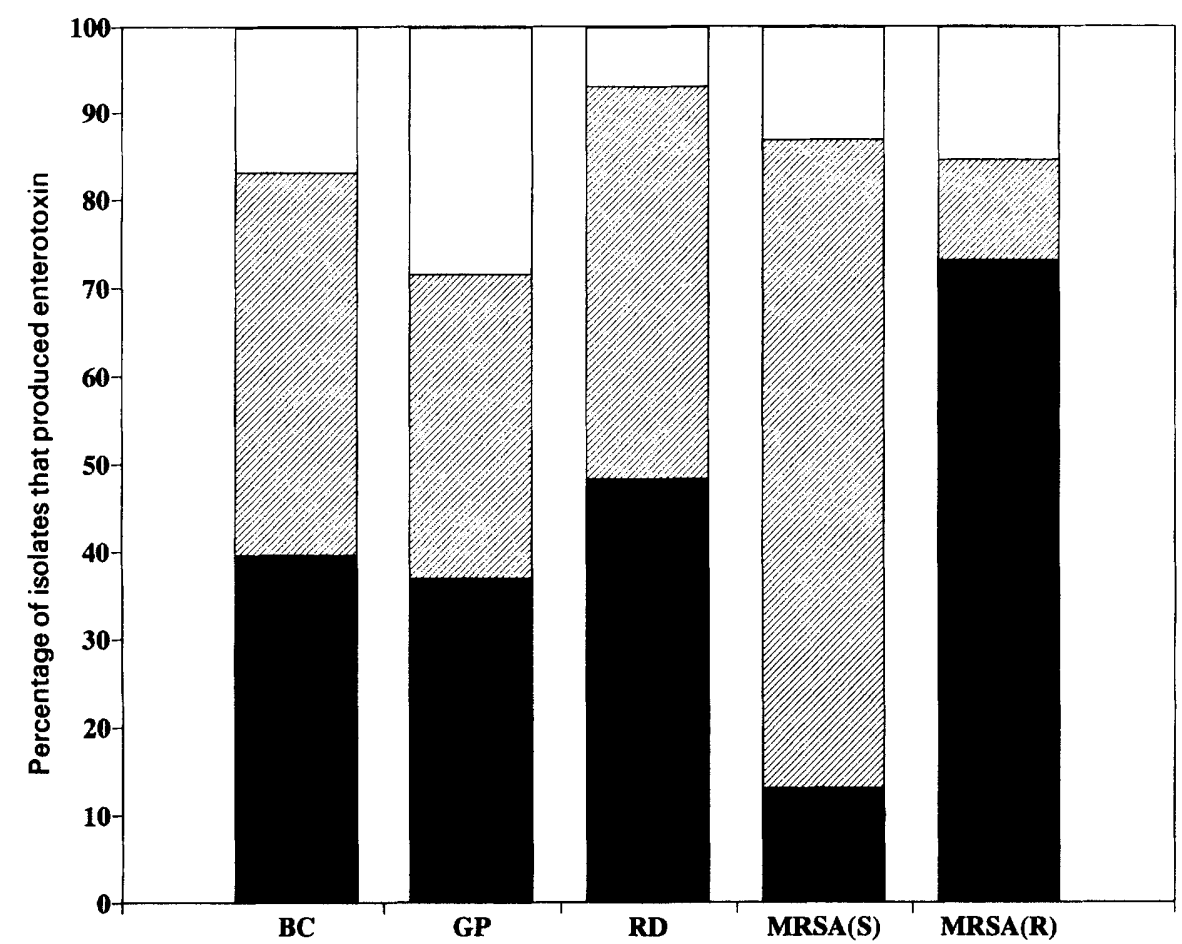

Fig. 2. Distribution of multiple enterotoxin production by $S$. aureus isolates from different sources: no enterotoxin $\square$; 1 enterotoxin $[\geq 2$ enterotoxins $\square$. 


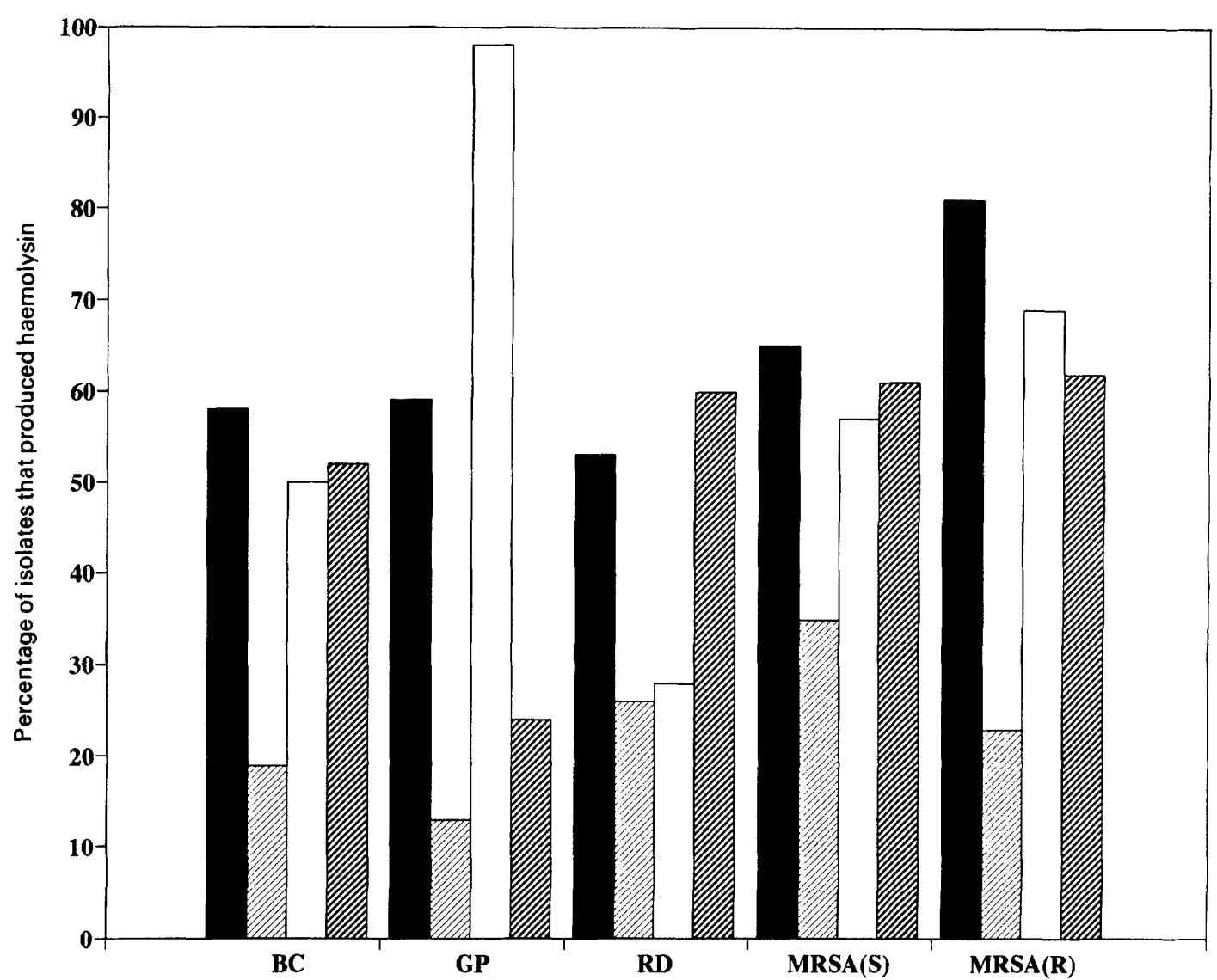

Fig. 3. Results of tests for haemolysins of $S$. aureus: $\alpha \square ; \beta \square ; \gamma \square ; \delta \square$

production amongst the MSSA and MRSA sub-groups $(p>0.5)$ and there was no difference in the relative combined total numbers of $\beta$-haemolysin-producing MSSA isolates (30 of 152) and MRSA isolates (14 of 49) $(p>0.5)$.

Considerably more GP isolates produced $\gamma$-haemolysin than did strains from either RD or BC collections ( $p<0 \cdot 001$ ). A less marked, but nonetheless significant increase was also apparent in the number of $\gamma$ haemolysin-producers amongst the $\mathrm{BC}$ isolates compared with the RD isolates of MSSA $(p<0.001)$. The proportion of $\gamma$-haemolysin producers in the aminoglycoside-sensitive and -resistant MRSA isolates did not differ significantly ( $\mathrm{p}>0.5$ ). Of MRSA isolates, 31 $(63 \%)$ of 49 were $\gamma$-haemolysin producers, a number similar to that of the BC MSSA isolates $(p>0.5)$, but

Table IV. Distribution of haemolysin production in isolates from different sources

\begin{tabular}{|c|c|c|c|c|c|}
\hline \multirow{2}{*}{$\begin{array}{l}\text { S. aureus } \\
\text { group }\end{array}$} & \multirow{2}{*}{$\begin{array}{l}\text { Number of } \\
\text { isolates }\end{array}$} & \multicolumn{4}{|c|}{$\begin{array}{l}\text { Number }(\%) \text { of isolates that } \\
\text { produced haemolysin }\end{array}$} \\
\hline & & $\alpha$ & $\beta$ & $\gamma$ & $\delta$ \\
\hline $\mathrm{BC}$ & 48 & $28(58)$ & $9(19)$ & $24(50)$ & $25(52)$ \\
\hline GP & 46 & $27(59)$ & $6(13)$ & $45(98)$ & $11(24)$ \\
\hline RD & 58 & $31(53)$ & $15(26)$ & $16(28)$ & $35(60)$ \\
\hline MRSA(S) & 23 & $15(65)$ & $8(35)$ & $13(57)$ & $14(61)$ \\
\hline MRSA(R) & 26 & $21(81)$ & $6(23)$ & $18(69)$ & $16(62)$ \\
\hline
\end{tabular}

somewhat higher and lower than the RD and GP MSSA isolates respectively ( $\mathrm{p}<0.001)$.

Significantly fewer GP than either BC or RD MSSA isolates produced $\delta$-haemolysin $(\mathrm{p}<0.001)$. The BC and $R D$ collections did not differ in this respect $(p>$ $0 \cdot 5$ ). Similarly, the aminoglycoside-sensitive and -resistant MRSA isolates did not differ significantly $(p>0.5)$. When the combined MRSA results (30 of 49) were compared with the combined $B C$ and $R D$ figures (60 of 106), representing "hospital isolates" of MSSA, there was no significant difference. However, it was clear that more MRSA isolates produced $\delta$ haemolysin than did GP MSSA isolates $(\mathrm{p}<0 \cdot 001)$.

The isolates were divided into haemolysin producers, defined as any isolate that produced one or more haemolysin, and non-producers. There was no significant difference between the numbers of haemolysinproducers in the various MSSA collections ( $p>0.5$ ) (table V); the MRSA collections were similarly homogeneous. The relative total numbers of haemolysin producing MSSA isolates (145 of 152) and MRSA isolates (44 of 49) did not differ significantly ( $p>0.5)$.

When the number of MSSA isolates in each of the BC, GP and RD sub-groups that produced less than two, two, or three or more haemolysins was compared, no significant differences could be demonstrated (table VI) $(p>0.5)$. Similarly, there was no significant difference between the aminoglycoside-resistant and -sensitive MRSA sub-groups ( $p>0.5$ ). Because the number of MRSA isolates that produced less than two 
Table V. Comparison of numbers of haemolysin producers and non-producers amongst isolates from different sources

\begin{tabular}{|c|c|c|c|}
\hline \multirow{2}{*}{$\begin{array}{l}\text { S. aureus } \\
\text { group }\end{array}$} & \multirow{2}{*}{$\begin{array}{l}\text { Number of } \\
\text { isolates }\end{array}$} & \multicolumn{2}{|c|}{$\begin{array}{c}\text { Number }(\%) \text { of isolates that were } \\
\text { haemolysin }\end{array}$} \\
\hline & & producers & non-producers \\
\hline $\mathrm{BC}$ & 48 & $47(98)$ & $1(2)$ \\
\hline GP & 46 & $45(98)$ & $1(2)$ \\
\hline RD & 58 & $53(91)$ & $5(9)$ \\
\hline MRSA(S) & 23 & $20(87)$ & 3 (13) \\
\hline MRSA(R) & 26 & $24(92)$ & $2(8)$ \\
\hline
\end{tabular}

haemolysins was small, and in order to facilitate a valid statistical comparison, isolates that produced two haemolysins were combined with those producing less than two haemolysins. The distributions of numbers of haemolysins produced by MSSA and MRSA isolates differed (fig. 4); the MRSA isolates produced significantly more haemolysins $(\mathrm{p}<0.001)$.

\section{Discussion}

If the pathogenicity of $S$. aureus is related to the production of enterotoxins it might be expected that isolates from "serious" infections, such as septicaemia, would be more likely to yield enterotoxins. Humphreys and co-workers in Dublin have shown previously that more than $63 \%$ of 52 blood culture isolates studied produced enterotoxins $\mathrm{A}, \mathrm{B}, \mathrm{C}$ or $\mathrm{D}$, alone or in combination, whereas only $11 \%$ of 27 nasal carriage isolates yielded enterotoxin (in every case enterotoxin $\mathrm{D}$ alone) ${ }^{20}$ In the present study $29(60 \%)$ of 48 septicaemia (BC) isolates produced enterotoxins. This finding is in close agreement with their experience and the distribution of individual enterotoxins was similar. However, the findings for the nasal carriage isolates were strikingly different: $29(63 \%)$ of 46 Glasgow isolates produced at least one enterotoxin, although, as in Dublin, this was usually enterotoxin D.

The marked disparity in the ability of nasal carriage isolates to produce enterotoxin was intriguing. Firstly, it demonstrates that nasal carriage isolates from geographically distinct locations do not express this characteristic homogeneously, and underlines the need to establish an appropriate local representative collection of organisms when making comparisons. Secondly, it suggests that even if enterotoxin production is associated with enhanced pathogenicity, the ability of $S$. aureus to produce disease depends on other factors as well.

The proportion of enterotoxin producers amongst the RD $S$. aureus isolates $(52 \%)$ was slightly lower than amongst the $\mathrm{BC}$ and $\mathrm{GP}$ isolates $(60 \%$ and $63 \%$ respectively), but not significantly so. The distribution of the individual enterotoxins was again similar. However, Jordens and co-workers did not detect enterotoxin production in any of 10 clinical isolates of MSSA from the London Hospital. ${ }^{17}$

It is not surprising that significant enterotoxin production is common in other clinical isolates. Many of the septicaemic episodes were hospital-acquired and the organisms involved would form a subset of those found in other clinical lesions from patients within the hospital. The higher proportion of $\mathrm{BC}$ isolates that produced enterotoxins, when compared with other strains, might have attained statistical

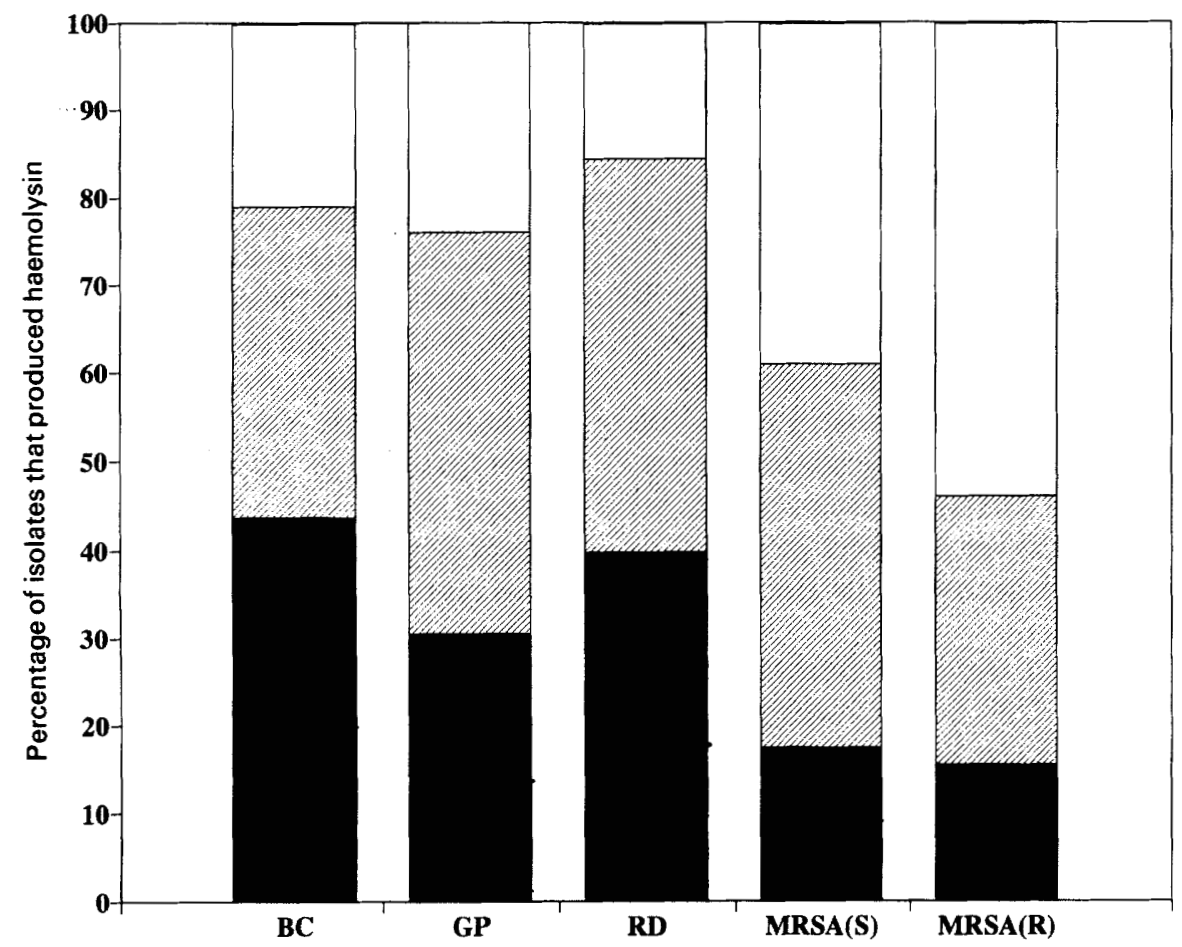

Fig. 4. Distribution of multiple haemolysin production by $S$. aureus isolates from different sources: $<2$ haemolysins $\square ; 2$ haemolysins $\geq$ 3 haemolysins $\square$. 
Table VI. Comparison of multiple haemolysin production by isolates from different sources

\begin{tabular}{cc|rrr}
\hline & & \multicolumn{3}{|c}{$\begin{array}{c}\text { Number(\%) of isolates that } \\
\text { produced }\end{array}$} \\
$\begin{array}{ccrrr}\text { S. aureus } \\
\text { group }\end{array}$ & $\begin{array}{c}\text { Number of } \\
\text { isolates }\end{array}$ & \multicolumn{3}{|c}{} \\
\cline { 3 - 5 } & & $<2^{*}$ & $2 *$ & $\geq 3^{*}$ \\
\hline BC & 48 & $21(44)$ & $17(35)$ & $10(21)$ \\
GP & 46 & $14(30)$ & $21(46)$ & $11(24)$ \\
RD & 58 & $23(40)$ & $26(45)$ & $9(15)$ \\
MRSA(S) & 23 & $4(17)$ & $10(44)$ & $9(39)$ \\
MRSA(R) & 26 & $4(15)$ & $8(31)$ & $14(54)$ \\
\hline
\end{tabular}

* Number of haemolysins.

significance if the sample size had been larger, and enterotoxin production may be related to the invasive capabilities of these organisms. Conversely, it could be argued that other factors, unrelated to enterotoxin production, are of greater importance in determining the ability of an organism to produce septicaemia. None of the patients whose organisms made up the RD collection subsequently developed $S$. aureus septicaemia.

The high rates of enterotoxin production by aminoglycoside-sensitive MRSA in GRI reported in this study were similar to those reported elsewhere ${ }^{17,19}$ $19(83 \%)$ of 23 isolates produced enterotoxin A; two of these isolates also produced enterotoxins B and C, and a third elaborated enterotoxins $B$ and $C$ only. However, $19(73 \%)$ of 26 aminoglycoside-resistant MRSA did not produce enterotoxins; three isolates produced enterotoxin $\mathrm{A}$ alone, two isolates enterotoxins $\mathrm{B}$ and $\mathrm{C}$, one isolate enterotoxins $\mathrm{A}, \mathrm{B}$ and $\mathrm{C}$, and one isolate enterotoxins $\mathrm{A}$ and $\mathrm{C}$.

In view of their cytotoxic effects, it might be expected that isolates from septicaemic episodes would be more likely to elaborate haemolysins. Humphreys ${ }^{19}$ showed that in such isolates from Dublin hospitals, the frequencies of $\alpha-, \gamma$ - and $\delta$-haemolysin production were in excess of $80 \%$. In the current study the corresponding figures were considerably lower. The frequencies of $\alpha$-and $\gamma$-haemolysin production $(58 \%$ and $50 \%$ respectively) were in closer agreement with those of Christenssen and Hedstrom ${ }^{25}$ who found $58 \%$ and $24 \%$ production amongst 88 septicaemia isolates of $S$. aureus. Whereas the Dublin group found little difference in the frequency of haemolysin production in septicaemia and nasal carriage isolates, we found substantially higher and lower numbers of $\gamma$ - and $\delta$ haemolysin producers respectively, in the carriage group.

With the exception of a very low frequency of $\gamma$ haemolysin production $(28 \%)$, the distribution of haemolysin production in the RD group was similar to that in the hospital septicaemic group, which again may reflect the fact that the $\mathrm{BC}$ isolates form a subset of the RD collection.

Frequencies of haemolysin production, and particularly multiple haemolysin production, tended to be higher in MRSA isolates from GRI than in other hospital isolates, but the numbers of $\alpha-(73 \%), \gamma-(63 \%)$ and $\delta-(61 \%)$ haemolysin producers were not as high as those found by Jordens et al. ${ }^{17}$ or Humphreys et al. ${ }^{19}$ In contrast with these studies, $28 \%$ of GRI MRSA isolates produced $\beta$-haemolysin. Unlike the findings with regard to enterotoxin production, there were no marked differences in the frequencies of haemolysin production in the aminoglycoside-sensitive and -resistant MRSA groups.

Clearly the results of tests for pathogenicity factors in $S$. aureus demonstrated a number of similarities with previously published data, as well as some important differences. The major differences concern the GP MSSA and the MRSA results. The GP MSSA results suggest that the relationship between enterotoxin and haemolysin production and pathogenicity is not simple. Enterotoxin production frequencies were very similar to, and haemolysin production frequencies only marginally lower than, the frequencies in their counterparts from clinical infection episodes; enterotoxin $\mathrm{C}$ and $\gamma$-haemolysin frequencies were significantly higher. Amongst the MRSA strains, the findings with the aminoglycoside-sensitive isolates were very similar to those with MRSA strains from elsewhere; high frequencies of enterotoxin A production were coupled with high frequencies of haemolysin production. However, whereas the aminoglycoside-resistant MRSA strains were indistinguishable with regard to haemolysin production, their very low frequency of enterotoxin production was quite different, and has not been described in MRSA strains from elsewhere. ${ }^{17,19}$

We have shown previously that MRSA isolates in GRI can be subdivided into two major groups on the basis of phage type, biotype, immunoblot and REFP data. ${ }^{22}$ To some extent these differences are reflected in the distribution of these organisms within discrete clinical areas of the hospital. The subdivision of GRI MRSA strains into enterotoxin A-producing aminoglycoside-sensitive isolates and predominantly enterotoxin non-producing aminoglycoside-resistant strains, as revealed by this study, mirrors the subgroups defined by the other methods (table VII). These results reinforce the hypothesis that MRSA strains in GRI are derived from two predominant clones.

There is little doubt that the enterotoxins and haemolysins of $S$. aureus are toxins of considerable potency. Equally, in a situation where all three MSSA groups produced these toxins with similar frequency, there must be other factors that determined whether an individual's experience of the organism resulted in asymptomatic carriage or fatal septicaemia. These factors would have included other toxins and enzymes produced by the organisms, opportunity for deeper tissue invasion, humoral and cellular immune status, nutritional status, age and underlying disease of the patient. This multitude of variables confounds attempts to understand pathogenicity in terms of properties of the organism alone.

To some extent animal studies and in-vitro systems 
Table VII. Summary of typical properties of aminoglycoside-sensitive and -resistant MRSA in Glasgow Royal Infirmary

\begin{tabular}{|c|c|c|c|c|c|}
\hline $\begin{array}{l}\text { Aminoglycoside } \\
\text { sensitivity }\end{array}$ & $\begin{array}{l}\text { Phage type } \\
\text { (RTD) }\end{array}$ & Biotype & $\begin{array}{l}\text { Immunoblot } \\
\text { pattern }\end{array}$ & REFP & Enterotoxin A \\
\hline & $\begin{array}{c}53 / 85+ \\
\text { "MRSA" phages* }\end{array}$ & $A$ or $C$ & Blot 1 & FP 1 & Producer \\
\hline Resistant & Non-typable & B or D & Blot 2 & FP 2 & Non-producer \\
\hline
\end{tabular}

* Supplementary phages for testing MRSA.

can help us to standardise the relative pathogenic potential of organisms. In the long term, it must be hoped that such studies in conjunction with an increased knowledge of immune mechanisms, and quantitation of those factors which render an individual susceptible to infection, will allow us to relate the

\section{References}

1. Adlam C, Easmon CSF. Immunity and hypersensitivity to staphylococcal infection. In: Staphylococci and staphylococcal infections, vol 1; clinical and epidemiological aspects. London, Academic Press. 1983: 275-323.

2. Shanson DC. Staphylococcal infections in hospital. Br J Hosp Med 1986; 35: 312-320

3. Musher DM, Verner EF. Treatment of infections due to Staphylococcus aureus. In: Jeljaszewicz J (ed) The staphylococci. Zbl Bakteriol, Mikrobiol Hyg 1985; Suppl 14: 407419.

4. Waldvogel FA. Treatment of infections due to methicillinresistant Staphylococcus aureus. J Hosp Infect 1986; 7 Suppl A: $37-46$.

5. Marples RR, Cooke EM. Current problems with methicillinresistant Staphylococcus aureus. J Hosp Infect 1988; 11 : 381-392.

6. Pavillard R, Harvey K, Douglas D et al. Epidemic of hospitalacquired infection due to methicillin-resistant Staphylococcus aureus in major Victorian hospitals. Med J Aust 1982; 1: $451-454$.

7. Turnidge J, Lawson $P$, Munro R, Benn R. A national survey of antimicrobial resistance in Staphylococcus aureus in Australian teaching hospitals. Med J Aust 1989; 150: 69-72.

8. Locksley RM, Cohen ML, Quinn TC et al. Multiply antibioticresistant Staphylococcus aureus: introduction, transmission and evolution of nosocomial infection. Ann Intern Med 1982;97: 317-324.

9. Cafferkey MT, Coleman DC, McGrath B et at. Methicillinresistant Staphylococcus aureus in Dublin 1971-84. Lancet $1985 ; 2: 705-708$.

10. Morgan MG, Harte-Barry MJ. Methicillin-resistant Staphylococcus aureus: a ten-year survey in a Dublin hospital. $J$ Hosp Infect 1989; 14: 357-362.

11. Marples RR. Methicillin-resistant Staphylococcus aureus. Curr Opin Infect Dis 1988; 1 : 722-726.

12. Casewell MW. Epidemiology and control of the 'modern' methicillin-resistant Staphylococcus aureus. J Hosp Infect 1986; 7 Suppl A: 1-11.

13. Maple PAC, Hamilton-Miller JMT, Brumfitt W. World-wide properties of organisms to the disease produced in patients.

This work was supported by a grant from the Scottish Hospitals Endowments Research Trust. We thank Professor J. D. Sleigh for encouragement and advice.

antibiotic resistance in methicillin-resistant Staphylococcus aureus. Lancet $1989 ; 1$ : 537-540.

14. Lacey RW. Multi-resistant Staphylococcus aureus-a suitable case for inactivity? J Hosp Infect 1987; 9: 103-105.

15. Lacey RW, Barr KW, Barr VE, Inglis TJ. Properties of methicillin-resistant Staphylococcus aureus colonizing patients in a burns unit. $J$ Hosp Infect 1986; 7: 137-148.

16. Roberts JIS, Gaston MA. Protein A and coagulase expression in epidemic and non-epidemic Staphylococcus aureus. $J$ Clin Pathol 1987; 40: 837-840.

17. Jordens JZ, Duckworth GJ, Williams RJ. Production of "virulence factors" by "epidemic" methicillin-resistant Staphylococcus aureus in vitro. J Med Microbiol 1989; 30: 245-252.

18. Cookson B, Talsania H, Naidoo J, Phillips I. Strategies for typing and properties of epidemic methicillin-resistant Staphylococcus aureus. Eur J Clin Microbiol 1986; 5: 702709.

19. Humphreys H, Keane CT, Hone R et al. Enterotoxin production by Staphylococcus aureus isolates from cases of septicaemia and from healthy carriers. J Med Microbiol $1989 ; 28$ : 163 172.

20. Duckworth GJ, Oppenheim BA. Enterotoxin production in epidemic methicillin-resistant Staphylococcus aureus. Lancet $1986 ; 1$ : 565-566.

21. Coia JE, Noor-Hussain I, Platt DJ. Plasmid profiles and restriction enzyme fragmentation patterns of plasmids of methicillin-sensitive and methicillin-resistant isolates of Staphylococcus aureus from hospital and the community. $J$ Med Microbiol 1988; 27 : 271-276.

22. Coia JE, Thomson-Carter F, Baird D, Platt DJ. Characterisation of methicillin-resistant Staphylococcus aureus by biotyping, immunoblotting and restriction enzyme fragmentation patterns. J Med Microbiol 1990; 31 : 125-132

23. Chao L-P, Birkbeck TH. Assay of staphylococcal $\delta$-haemolysin with fish erythrocytes. J Med Microbiol 1978; 11: 303-313.

24. Siegel S. Non-parametric statistics for the behavioural sciences, London, McGraw-Hill. 1956: 104-111.

25. Christensson B, Hedström SA. Biochemical and biological properties of Staphylococcus aureus septicaemia strains in relation to clinical characteristics. Scand J Infect Dis 1986; 18: $297-303$ 